ankylosing spondylitis (AS), suggesting a role for HLA-B27 misfolding in the pathogenesis of the human disease and the UPR has been linked to IL23 production.

Aim of the Study To study endoplasmic reticulum stress and activation of the UPR ex vivo in the synovium and peripheral blood mononuclear cells (PBMCs) of HLA-B27 positive AS patients as compared to patients with other inflammatory joint diseases and healthy controls.

Materials and Methods Synovial tissues were obtained from actively inflamed knees from patients with AS (HLA-B27 positive), other forms of spondyloarthritis (SpA) (HLA-B27 positive or negative), rheumatoid arthritis (RA) (HLA-B27 positive or negative) and other inflammatory joint diseases ("non $\mathrm{SpA} / \mathrm{RA}$ inflammatory joint disease') (HLA-B27 positive or negative) ( $n=10$ in each group). PBMCs were isolated from whole blood samples taken from patients with AS (HLA-B27 positive), RA (HLA-B27 negative) and healthy controls (HLA-B27 negative) ( $\mathrm{n}=12$ in each group). Activation of the UPR in all samples was analysed using quantitative RT-PCR with primers for DDIT3 (also known as CHOP), HSPA5 (also known as BiP), DNAJB9 and XBP1s. Gene expression levels were correlated to disease activity scores such as DAS28, ASDAS, BASDAI as well as to CRP levels.

Results None of the UPR associated genes was specifically upregulated in either the blood cells or synovial tissue from AS patients as compared to the other groups. In the synovial samples, only DDIT3 expression was found significantly different between healthy controls and HLA B27 negative SpA patients on the one hand and non$\mathrm{SpA} / \mathrm{RA}$ inflammatory joint disease on the other hand. There was no significant difference in expression of HSPA5, DNAJB9 and XBP1s between the different groups. In PBMCs, expression of DDIT3 was different between groups and levels were significantly lower in the AS group compared to the RA group and the healthy controls. There was no difference in expression of the other genes between the groups.

Discussion Our data do not provide support for a critical and unique role of the UPR in AS and other forms of SpA. The difference in expression of DDIT3 in PBMCs with an increase in RA patient samples compared to AS patient samples suggests specific involvement of this gene in RA.

\section{A10.12 CHARACTERISATION OF A NEW mPGES-1 INHIBITOR IN HUMAN AND MURINE MODELS OF INFLAMIMATION}

doi:10.1136/annrheumdis-2013-203224.12

'Patrick Leclerc, 'Helena Idborg, ${ }^{2}$ Patrick Stenberg, 'Marina Korotkova, 'Per-Johan Jakobsson. 'Rheumatology Research Unit, CMM, Karolinska Institute, Stockholm, Sweden; ${ }^{2}$ NovaSAID $A B$

Background Microsomal prostaglandin E synthase-1 (mPGES-1) inhibition has been suggested as an alternative to cyclooxygenase (COX) inhibition in the treatment of pain and inflammation. This approach could potentially mitigate the gastro-intestinal and cardiovascular side effects associated to traditional non-steroidal antiinflammatory drugs (NSAIDs) and Coxibs.

Aim To characterise a selective inhibitor of mPGES-1 and study its impact on the prostanoid profile in various models of inflammation.

Materials and Methods Potency and selectivity: Compound III was incubated with active enzymes of the prostanoid pathway (mPGES-1, COX-1/2, PGIS, H-PGDS). For potential cross-reactivity towards thromboxane synthase, compound III was tested in a thromboxane release assay in platelets. A549 cells: compound III was assayed in both short and long-term assays. Cells were stimulated with IL-1 $\beta /$ TNF- $\alpha$ or IL-1 $\beta$ alone and incubated in the presence of inhibitors. Whole blood assay/mouse peritoneal macrophages: blood and macrophages were induced with LPS and incubated with compound III for $24 \mathrm{~h}$. Air pouch model: the inflammatory reaction was triggered by a carrageenan injection intrapouch. Inhibitors were injected i.p. and exudates were collected after $6 \mathrm{~h}$. Prostanoid analysis: Prostanoids were measured by mass spectrometry or enzyme immunoassay.

Results Compound III has an IC $_{50}$ of $0.4 \mu \mathrm{M}$ and $1 \mu \mathrm{M}$ in human and murine mPGES-1 respectively. It has no activity on other enzymes of the prostanoid pathway and did not inhibit thromboxane release from human platelets. In cellular assays, it reduced $\mathrm{PGE}_{2}$ production in A549 cells, mouse peritoneal macrophages and LPSstimulated whole blood. In mouse peritoneal macrophages, compound III caused a shunt to the prostacyclin pathway. Lastly, we assayed compound III in the air pouch model to verify its impact on the prostanoid profile and compare it to the profile obtained in mPGES-1 k.o. mice. As opposed to mPGES-1 genetic deletion, which attenuated $\mathrm{PGE}_{2}$ induction and caused a shunt to the thromboxane pathway, mPGES-1 inhibition with compound III reduced $\mathrm{PGE}_{2}$ production and tended to decrease the levels of other prostanoids.

Conclusions Compound III is an active and selective inhibitor of mPGES-1. Its impact on the prostanoid profile was assay dependent. However, in the air pouch, contrary to mPGES-1 gene deletion, compound III did not trigger shunting, a scenario more likely to represent the outcome of mPGES-1 inhibition at the inflammatory site.

\section{A10.13 IDENTIFICATION OF IMMUNOPHENOTYPIC SIGNATURES IN PERIPHERAL BLOOD OF MULTIPLE SCLEROSIS PATIENTS BY MULTIPARAMETRIC FLOW CYTOMETRY}

doi:10.1136/annrheumdis-2013-203224.13

'Ursula Schulte-Wrede, ${ }^{2}$ Berit Rosche, 'Joachim R Grün, 'Andreas Radbruch, 'Andreas Grützkau. 'Deutsches Rheuma-Forschungszentrum (DRFZ), Ein Leibniz-Institut, ImmuneMonitoring Core Facility, Berlin, Germany; ${ }^{2}$ Charité-Universitätsmedizin der HU-Berlin, Abteilung für Neurologie, Berlin, Germany

Background Multiple Sclerosis (MS) is a chronic inflammatory and demyelinating disease of the CNS with unknown aetiology until now. CD4+ T helper (Th) 1 cells, proinflammatory Th17 cells, CD8 + T cells, B cells, natural killer (NK) cells and denritic cells (DC) are accepted to play in important role in pathogenesis of disease. Lymphocytes of the peripheral blood from multiple sclerosis (MS) patients are characterised by proinflammatory function but robust cell surface markers to distinguish patients from controls are not available until now.

Methods/Results In this study we analysed frequency and phenotype of blood cell subsets by multicolour staining including up to 50 different monoclonal antibodies that allowed detecting 894 parameter combinations per sample, including 99 control parameters. Relative event numbers, absolute cell numbers and relative fluorescence intensities of all fluorochromes were compared between 10 patients with a clinically isolated syndrome (CIS) or early relapsing-remitting MS (RRMS) and 10 healthy age- and sexmatched controls and were analysed in a semiautomatic manner. Statistical significant results in expression profile between both groups were found involved receptors in inflammation (CD119, CD62L, CRTH2) on cells of adaptive and also innate immunity (Welch t-test <0.05).

Conclusions The results identify a MS specific profile of peripheral blood leukocytes by a multiparametric cytometric approach. Thus, the large-scale immunophenotyping is appropriate tool to identify new disease relevant leukocyte subtypes and receptors and may have implications for diagnosis, pathophysiological understanding and therapy-monitoring of patients with MS and other autoimmune diseases. 